\title{
Risk factors affecting the 1-year outcomes of minor ischemic stroke: results from Xi'an stroke registry study of China
}

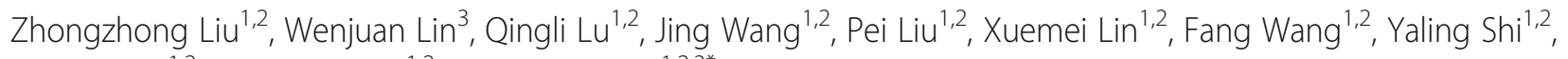
Qing Wang ${ }^{1,2}$, Guozheng Liu ${ }^{1,2}$ and Songdi $\mathrm{Wu}^{1,2,3^{*}}$ (i)

\begin{abstract}
Background: The prevalence of stroke recurrence, disability, and all-cause mortality of patients with minor ischemic stroke (MIS) remains problematic. The aim of the present study was to identify risk factors associated with adverse outcomes at 1 year after MIS in the Xi'an region of China.

Methods: This prospective cohort study included MIS patients above 18 years old with National Institutes of Health Stroke Scale (NIHSS) score $\leq 3$ who were treated in any of four hospitals in Xi'an region of China between January and December 2015. The 1-year prevalence of stroke recurrence, disability, and all-cause mortality were evaluated, respectively. Multivariate logistic regression analysis was performed to assess the association between the identified risk factors and clinical outcomes.

Results: In this study, 131(10.5\%, 131/1252) patients were lost to follow-up at 1 year. A total of 1121 patients were included for analysis, the prevalence of stroke recurrence, disability, and all-cause mortality at 1 year after MIS were 3.4\% (38/1121), 9.3\% (104/1121), and 3.3\% (37/1121), respectively. Multivariate logistic regression analysis identified age, current smoking, and pneumonia as independent risk factors for stroke recurrence. Age, pneumonia, and alkaline phosphatase were independent risk factors for all-cause mortality. Independent risk factors for disability were age, pneumonia, NIHSS score on admission, and leukocyte count.

Conclusions: The 1-year outcomes of MIS in Xi'an region of China were not optimistic, especially with a high prevalence of disability. The present study indicated that age and pneumonia were the common independent risk factors affecting the 1-year outcomes of MIS in Xi'an region of China.
\end{abstract}

Keywords: Risk factors, Minor ischemic stroke, Stroke recurrence, Disability, All-cause mortality

\section{Background}

The prevalence of stroke has significantly increased over the last two decades worldwide [1]. The Global Burden of Disease Study 2010 reported approximately 1.7 million deaths by stroke, which has become the leading cause of

\footnotetext{
* Correspondence: wusongdi@gmail.com

'The First Affiliated Hospital of Northwest University, Xi'an 710069, Shaanxi, China

${ }^{2}$ Department of Neurology, The First Hospital of Xi'an, No.30, Fenxiang Road, South Street, Xi'an 710002, Shaanxi, China

Full list of author information is available at the end of the article
}

death and adult disability in China [2, 3]. The high recurrence and disability rates seriously affect the health and quality of life of stroke patients. A minor ischemic stroke (MIS) is defined as a National Institutes of Health Stroke Scale (NIHSS) score of $\leq 3$ [4]. A MIS is typically treated conservatively with antiplatelet agents and general strategies for the prevention of vascular injury. Yet, the rate of recurrent stroke and progression of stroke remain high, as up to one in four of MIS patients are disabled or died at follow-up [5]. Therefore, it is critical to identify risk 
factors for stroke recurrence, disability, and all-cause mortality associated with MIS, as most previous studies had focused on long-term risk factors and early detection, while data regarding regional and 1-year risk factors for poor clinical outcomes are relatively limited [6, 7]. Moreover, there may be differences in risk factors for poor clinical outcomes of MIS patients among different countries, regions, and ethnicities. In this study, data regarding the prevalence and poor clinical outcomes of MIS from January to December 2015 were collected from four level 3 first-class hospitals in the Xi'an region of China to identify risk factors associated with stroke recurrence, disability, and all-cause mortality at 1 year after MIS.

\section{Methods}

\section{Study population}

This prospective cohort study included MIS patients aged $\geq 18$ years old who were treated at any of four hospitals in the Xi'an region of China from January to December 2015. Diagnosis of acute ischemic stroke by computed tomography (CT) or magnetic resonance imaging (MRI) according to world Health Organization criteria [8]. The NIHSS score on admission was used to assess the initial neurological severity of the patients [4, 9]. MIS is defined as a NIHSS score at admission $\leq 3$ [4]. Patients diagnosed with MIS due to non-vascular causes (primary and meta-static neoplasms, paralysis after seizure, traumatic brain injury, etc.) that led to a brain function deficit or intracerebral hemorrhage, as determined by CT or MRI, were excluded from analysis, as were those with incomplete follow-up data at 1 year after MIS onset. The diagnostic criteria of all participating hospitals were consistent.

\section{Data collection}

Baseline informations were collected from the four level 3 first-class hospitals participating in this study within $24 \mathrm{~h}$ after admission, including demographic information, past medical history, admission evaluation, laboratory data and complications [10]. Peripheral vascular disease (PVD) was defined as intermittent claudication, an ankle brachial index $<0.9$, or history of intermittent claudication with relevant interventional therapies (lower limb artery angioplasty/bypass/other vascular interventional treatments/lower limb amputation). Body mass index (BMI) and other associated complications were defined in accordance with the Chinese National Stroke Registry study [6]. The NIHSS score was used to assess the severity of neurological impairment within $24 \mathrm{~h}$ of admission [11]. The occurrence of pneumonia during hospitalization was also recorded. All fasting blood samples were processed within $2 \mathrm{~h}$ of collection. Measurements of quality control specimens were conducted in a blinded manner in a central laboratory.

\section{Outcome assessment}

The patients were followed up at 1, 3, 6 and 12 months after MIS onset. All enrolled patients were interviewed face-to-face or contacted over the telephone by trained research coordinators. The interviewers were trained centrally with a standardized interview protocol and were blinded to a history of MIS for all patients [12]. The outcome events of 1-year follow-up in this study included stroke recurrence, disability and all-cause mortality. Stroke recurrence was defined as the occurrence of new acute stroke events during follow-up (including cerebral infarction, cerebral hemorrhage, subarachnoid hemorrhage), and the diagnosis of acute stroke were consistent with the World Health Organization definition stroke diagnostic criteria [13]. Confirmation of outcome events were sought from the treating hospital, and suspected stroke recurrence events without hospitalization were judged by independent outcome events judgement committee. Stroke disability was defined as a modified Rankin scale score $\geq 3$ (include death) at 1 year after MIS onset [14]. All-cause mortality was defined as death from any cause, as confirmed by either a death certificate from the medical record of the treating hospital or local citizen registry.

\section{Statistical analysis}

Continuous variables that conformed to a normal distribution were expressed as mean \pm standard deviation, and did not conform to a normal distribution were expressed as median (interquartile range). Categorical variables are reported as the frequency (\%). The chi-squared test (or Fisher's exact test, where appropriate) was used for comparison between groups, one-way analysis of variance (or Kruskal-Wallis test, where appropriate) for continuous variables. Univariate logistic regression analysis was used to identify baseline differences in clinical variables of patients with vs. without stroke recurrence, disability, and all-cause mortality. Multivariate logistic regression analysis was performed to analyze the associated affect factors of 1-year outcome events of MIS after adjustment for relevant covariates. Sensitive analysis was performed to analyze the influence between the loss to follow up patients and the clinical outcomes. All the estimates parameters were significant at $p<0.05$ level and the clinical significance were included in the multivariable logistic regression analysis. A two-tailed probability $(p)$ value of $<0.05$ was considered statistically significant. All analyses were performed with $\mathrm{R}$ statistical software (http://www.R-project.org; The R Foundation) and EmpowerStats (http://www.empowerstats.com; X\&Y Solutions, Inc., Boston, MA, USA).

\section{Results}

Patients recruitment

Of a total of 3117 patients who were initially enrolled in this study, 416 patients were excluded due to non-acute 
ischemic stroke in addition to 1449 with an NIHSS score at admission of $>3$. One thousand two hundred and fiftytwo patients had experienced minor ischemic stroke. Among these, 131(10.5\%, 131/1252) patients were lost to follow-up. Finally, a total of 1121 patients with MIS (initial NIHSS score $\leq 3$ ) were included for analysis (Fig. 1).

\section{Univariate analysis of patients with vs. without stroke recurrence, disability, and all-cause mortality at 1 year after MIS}

Among the 1121 patients included for analysis, the prevalence of stroke recurrence, disability, and all-cause mortality at 1 year after MIS was 3.4\% (38/1121), 9.3\% (104/1121), and $3.3 \%$ (37/1121), respectively (Tables 1,2 , and 3$)$. The results of univariate analysis showed that patients with stroke recurrence at 1 year after MIS were more likely to be older, current smokers, and had a history of previous stroke, PVD, and/or pneumonia than those without. There was no significant difference between patients with vs. without stroke recurrence in the sex ratio, BMI on admission, education level, medical insurance type, hypertension, DM, dyslipidemia, AF, moderate to heavy alcohol use, NIHSS score on admission, SBP and DBP on admission, heart rate, total cholesterol, triglycerides, HDL-C, LDL-C, FBG, blood urea nitrogen (BUN), uric acid (UA), alkaline phosphatase (ALP), blood platelet count (BPC), and leukocyte count (Table 1).
As shown in Table 2, older patients were more likely to be disabled at 1 year after MIS $(p<0.05)$ and those with a disability were significantly more likely to have a history of previous stroke and/or pneumonia, and a higher NIHSS score on admission, as well as higher BUN measurements and leukocyte count, as compared to those without. There was no significant difference between patients with vs. without disability in the sex ratio, BMI on admission, education level, medical insurance type, hypertension, DM, dyslipidemia, AF, smoking, moderate to heavy alcohol use, PVD, SBP and DBP on admission, heart rate, total cholesterol, triglyceride, HDL-C, LDL-C, FBG, UA, ALP, and BPC.

Factors associated with all-cause mortality in patients after MIS are shown in Table 3. The results of univariate analysis showed that the factors associated with death included age, PVD, pneumonia, and ALP (all $p<0.05$ ).

Regardless of whether the patients lost to follow-up were all considered to have no stroke recurrence, disability or all-cause mortality respectively, sensitivity analysis showed that the risk factors between the two groups with or without this part of patients were almost the same. There were only a few differences between the two groups when the patients lost to follow-up were all considered to be stroke recurrence, disability or allcause mortality, respectively. In addition, by comparing the clinical characteristics of two groups with or without

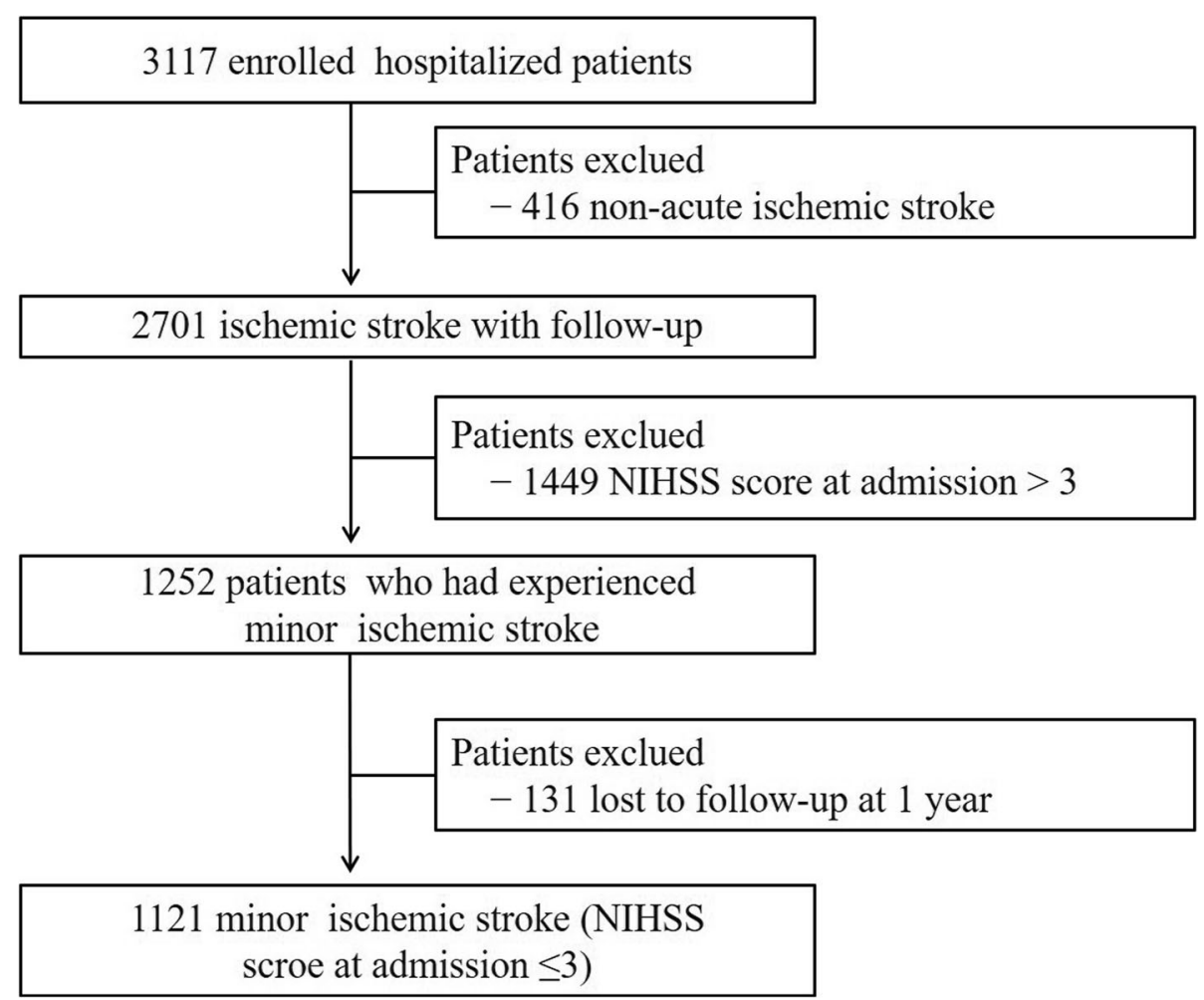

Fig. 1 Flow chart showing the patient selection. NIHSS, National Institutes of Health Stroke Scale 
Table 1 Univariate logistic regression analysis of patients with or without stroke recurrence at 1 year after MIS

\begin{tabular}{|c|c|c|c|c|c|}
\hline Variables & $\begin{array}{l}\text { Overall } \\
N=1121\end{array}$ & $\begin{array}{l}\text { Recurrence } \\
N=38(3.4 \%)\end{array}$ & $\begin{array}{l}\text { No recurrence } \\
N=1083(96.6 \%)\end{array}$ & OR $(95 \% \mathrm{Cl})$ & $P$ value \\
\hline \multicolumn{6}{|l|}{ Demographic information } \\
\hline Age, median (IQR), year & $64.0(56.0-74.0)$ & $70.0(61.2-76.8)$ & $64.0(55.0-73.0)$ & $1.05(1.02-1.08)$ & 0.001 \\
\hline Male, n(\%) & $697(62.2)$ & $29(76.3)$ & $668(61.7)$ & $1.97(0.93-4.17)$ & 0.075 \\
\hline BMl on admission, $\mathrm{kg} / \mathrm{m}^{2}$ & $23.9(22.2-25.6)$ & $24.1(22.6-26.1)$ & $23.9(22.1-25.6)$ & $1.00(0.90-1.10)$ & 0.935 \\
\hline Education level, n(\%) & & & & & 0.068 \\
\hline elementary or below & $446(39.8)$ & $18(47.4)$ & $428(39.5)$ & Ref. & - \\
\hline middle school & $234(20.9)$ & $6(15.8)$ & $228(21.1)$ & $0.62(0.25-1.57)$ & 0.319 \\
\hline high school or above & $441(39.3)$ & $14(36.8)$ & $427(39.4)$ & $0.78(0.39-1.57)$ & 0.485 \\
\hline Medical insurance type, n(\%) & & & & & 0.636 \\
\hline Urban employees' medical insurance & $591(52.7)$ & $19(50.0)$ & $572(52.8)$ & Ref. & - \\
\hline New type rural cooperative medical system & $414(36.9)$ & $17(44.7)$ & $397(36.7)$ & $1.29(0.67-2.48)$ & 0.443 \\
\hline Commercial insurance & $2(0.2)$ & $0(0.0)$ & $2(0.2)$ & $0.00(0.00-\infty)$ & 0.996 \\
\hline Out-of-pocket medical & $114(10.2)$ & $2(5.3)$ & $112(10.3)$ & $0.55(0.13-2.35)$ & 0.418 \\
\hline Vascular risk factors & & & & & - \\
\hline Hypertension, n(\%) & $768(68.5)$ & $26(68.4)$ & $742(68.5)$ & $0.99(0.50-1.96)$ & 0.974 \\
\hline $\mathrm{DM}, \mathrm{n}(\%)$ & $242(21.6)$ & $12(31.6)$ & $230(21.2)$ & $1.68(0.85-3.32)$ & 0.139 \\
\hline Dyslipidemia, n(\%) & $77(6.9)$ & $1(2.6)$ & $76(7.0)$ & $0.36(0.05-2.65)$ & 0.318 \\
\hline$A F, n(\%)$ & $54(4.8)$ & $3(7.9)$ & $51(4.7)$ & $1.69(0.52-5.48)$ & 0.386 \\
\hline Smoking, $\mathrm{n}(\%)$ & & & & & 0.017 \\
\hline Never smoking & $665(59.3)$ & $12(31.6)$ & $653(60.3)$ & Ref. & - \\
\hline smoking cessation & $169(15.1)$ & $9(23.7)$ & $160(14.8)$ & $1.85(0.89-4.38)$ & 0.068 \\
\hline Current smoking & $287(25.6)$ & $17(44.7)$ & $270(24.9)$ & $1.24(1.11-2.96)$ & 0.034 \\
\hline Moderate or heavy alcohol use, n(\%) & $271(24.2)$ & $7(18.4)$ & $264(24.4)$ & $0.70(0.31-1.59)$ & 0.391 \\
\hline Previous stroke, n(\%) & $297(26.5)$ & $18(47.4)$ & $279(25.8)$ & $2.54(1.34-4.80)$ & 0.004 \\
\hline PVD, n(\%) & $29(2.6)$ & $3(7.9)$ & $26(2.4)$ & $3.35(1.03-10.91)$ & 0.044 \\
\hline Pneumonia, n(\%) & $25(2.2)$ & $6(15.8)$ & $19(1.8)$ & $9.89(4.13-23.68)$ & 0.001 \\
\hline NIHSS score on admission & $1.0(0.0-2.0)$ & $1.5(0.0-3.0)$ & $1.0(0.0-2.0)$ & $1.04(0.80-1.37)$ & 0.755 \\
\hline SBP on admission, mmHg & $144.8 \pm 20.7$ & $145.6 \pm 24.2$ & $144.8 \pm 20.6$ & $1.00(0.99-1.02)$ & 0.788 \\
\hline DBP on admission, mmHg & $85.7 \pm 12.2$ & $86.6 \pm 14.6$ & $85.7 \pm 12.1$ & $1.01(0.98-1.03)$ & 0.661 \\
\hline Heart rate, bmp & $74.3 \pm 9.4$ & $76.8 \pm 10.0$ & $74.2 \pm 9.3$ & $1.03(1.00-1.06)$ & 0.086 \\
\hline \multicolumn{6}{|l|}{ Laboratory findings } \\
\hline Total cholesterol, mmol/L & $4.4 \pm 1.0$ & $4.5 \pm 0.9$ & $4.4 \pm 1.0$ & $1.04(0.76-1.41)$ & 0.821 \\
\hline Triglyceride, mmol/L & $1.7 \pm 1.2$ & $1.6 \pm 0.9$ & $1.7 \pm 1.2$ & $0.91(0.65-1.26)$ & 0.560 \\
\hline $\mathrm{HDL}-\mathrm{C}, \mathrm{mmol} / \mathrm{L}$ & $1.1 \pm 0.3$ & $1.1 \pm 0.3$ & $1.1 \pm 0.3$ & $0.57(0.18-1.79)$ & 0.337 \\
\hline $\mathrm{LDL}-\mathrm{C}, \mathrm{mmol} / \mathrm{L}$ & $2.6 \pm 0.8$ & $2.8 \pm 0.7$ & $2.6 \pm 0.9$ & $1.19(0.84-1.68)$ & 0.332 \\
\hline $\mathrm{FBG}, \mathrm{mmol} / \mathrm{L}$ & $5.8 \pm 2.1$ & $6.2 \pm 3.0$ & $5.7 \pm 2.1$ & $1.09(0.97-1.22)$ & 0.169 \\
\hline BUN, mmol/L & $5.0 \pm 1.8$ & $5.3 \pm 1.7$ & $5.0 \pm 1.8$ & $1.08(0.93-1.26)$ & 0.321 \\
\hline UA, $10 \mu \mathrm{mol} / \mathrm{L}$ & $291.3 \pm 96.0$ & $277.9 \pm 95.8$ & $291.8 \pm 96.1$ & $0.98(0.95-1.02)$ & 0.387 \\
\hline$A L P, U / L$ & $77.6 \pm 25.6$ & $83.8 \pm 21.5$ & $77.3 \pm 25.7$ & $1.01(1.00-1.02)$ & 0.104 \\
\hline $\mathrm{BPC}, \times 10^{11} / \mathrm{L}$ & $194.4 \pm 58.3$ & $193.4 \pm 48.0$ & $194.4 \pm 58.6$ & $0.97(0.55-1.71)$ & 0.906 \\
\hline Leukocyte count, $\times 10^{9} / \mathrm{L}$ & $6.7 \pm 2.2$ & $7.1 \pm 2.5$ & $6.6 \pm 2.2$ & $1.08(0.95-1.21)$ & 0.234 \\
\hline
\end{tabular}

Abbreviations: BMI Body Mass Index, DM Diabetes Mellitus, AF Atrial Fibrillation, PVD Peripheral Vascular Disease, NIHSS National Institutes of Health Stroke Scale, SBP Systolic Blood Pressure, DBP Diastolic Blood Pressure, HLD-C High-Density Lipoprotein Cholesterol, LDL-C low-density lipoprotein cholesterol, FBG Fasting Blood Glucose, BUN Blood Urea Nitrogen, UA Uric Acid, ALP Alkaline Phosphatase, BPC Blood Platelet Count, Cl confidence internals, OR odds ratios, IQR interquartile range 
Table 2 Univariate logistic regression analysis of patients with or without stroke disability at 1 year after MIS

\begin{tabular}{|c|c|c|c|c|c|}
\hline Variables & $\begin{array}{l}\text { Overall } \\
\mathrm{N}=1121\end{array}$ & $\begin{array}{l}\text { Disability } \\
N=104(9.3 \%)\end{array}$ & $\begin{array}{l}\text { No disability } \\
N=1017 \text { (90.7\%) }\end{array}$ & OR $(95 \% \mathrm{Cl})$ & $P$ value \\
\hline \multicolumn{6}{|l|}{ Demographic information } \\
\hline Age, median (IQR), year & $64.0(56.0-74.0)$ & $76.0(66.0-80.2)$ & $63.0(55.0-72.0)$ & $1.08(1.06-1.10)$ & 0.001 \\
\hline Male, n\% & $697(62.2)$ & $71(68.3)$ & $626(61.6)$ & $1.32(0.87-1.99)$ & 0.189 \\
\hline BMl on admission, $\mathrm{kg} / \mathrm{m}^{2}$ & $23.9(22.2-25.6)$ & $23.7(21.6-25.2)$ & $24.0(22.2-25.6)$ & $0.94(0.88-1.00)$ & 0.058 \\
\hline Education level, n (\%) & & & & & 0.052 \\
\hline elementary or below & $446(39.8)$ & $52(50.0)$ & $394(38.7)$ & Ref. & - \\
\hline middle school & $234(20.9)$ & $11(10.6)$ & $223(21.9)$ & $0.40(0.21-1.06)$ & 0.055 \\
\hline high school or above & $441(39.3)$ & $41(39.4)$ & $400(39.3)$ & $0.79(0.52-1.19)$ & 0.254 \\
\hline Medical insurance type, n(\%) & & & & & 0.919 \\
\hline Urban employees' medical insurance & $591(52.7)$ & $53(51.0)$ & $538(52.9)$ & Ref. & - \\
\hline New type rural cooperative medical system & $414(36.9)$ & $41(39.4)$ & $373(36.7)$ & $1.12(0.75-1.69)$ & 0.573 \\
\hline Commercial insurance & $2(0.2)$ & $0(0.0)$ & $2(0.2)$ & $0.00(0.00-\infty)$ & 0.996 \\
\hline Out-of-pocket medical & $114(10.2)$ & $10(9.6)$ & $104(10.2)$ & $0.97(0.49-1.91)$ & 0.931 \\
\hline \multicolumn{6}{|l|}{ Vascular risk factors } \\
\hline Hypertension, n (\%) & $768(68.5)$ & $74(71.2)$ & $694(68.2)$ & $1.11(0.73-1.70)$ & 0.622 \\
\hline $\mathrm{DM}, \mathrm{n}(\%)$ & $242(21.6)$ & $27(26.0)$ & $215(21.1)$ & $1.27(0.82-1.97)$ & 0.289 \\
\hline Dyslipidemia, n (\%) & $77(6.9)$ & $6(5.8)$ & $71(7.0)$ & $0.81(0.36-1.86)$ & 0.625 \\
\hline$A F, n(\%)$ & $54(4.8)$ & $8(7.7)$ & $46(4.5)$ & $1.67(0.81-3.44)$ & 0.163 \\
\hline Smoking, n (\%) & & & & & 0.053 \\
\hline Never smoking & $665(59.3)$ & $65(62.5)$ & $600(59.0)$ & Ref. & - \\
\hline Smoking cessation & $169(15.1)$ & $22(21.2)$ & $147(14.5)$ & $1.37(0.85-2.23)$ & 0.198 \\
\hline Current smoking & $287(25.6)$ & $17(16.3)$ & $270(26.5)$ & $0.60(0.35-1.03)$ & 0.063 \\
\hline Moderate or heavy alcohol use, n (\%) & $271(24.2)$ & $19(18.5)$ & $198(19.5)$ & $0.89(0.69-1.32)$ & 0.010 \\
\hline Previous stroke, $\mathrm{n}(\%)$ & $297(26.5)$ & $40(38.5)$ & $257(25.3)$ & $1.78(1.20-2.64)$ & 0.004 \\
\hline PVD, n (\%) & $29(2.6)$ & $4(3.8)$ & $25(2.5)$ & $1.57(0.58-4.26)$ & 0.378 \\
\hline Pneumonia, n (\%) & $25(2.2)$ & $12(11.5)$ & $13(1.3)$ & $7.58(4.15-13.84)$ & 0.001 \\
\hline NIHSS score on dmission & $1.0(0.0-2.0)$ & $2.0(0.0-3.0)$ & $1.0(0.0-2.0)$ & $1.28(1.08-1.50)$ & 0.004 \\
\hline SBP on admission, $\mathrm{mmHg}$ & $144.8 \pm 20.7$ & $146.8 \pm 22.2$ & $144.6 \pm 20.6$ & $1.00(1.00-1.01)$ & 0.297 \\
\hline DBP on admission, $\mathrm{mmHg}$ & $85.7 \pm 12.2$ & $84.7 \pm 14.9$ & $85.8 \pm 11.9$ & $0.99(0.98-1.01)$ & 0.390 \\
\hline Heart rate, bmp & $74.3 \pm 9.4$ & $75.5 \pm 9.0$ & $74.1 \pm 9.4$ & $1.01(0.99-1.03)$ & 0.154 \\
\hline \multicolumn{6}{|l|}{ Laboratory findings } \\
\hline Total cholesterol, mmol/L & $4.4 \pm 1.0$ & $4.4 \pm 1.0$ & $4.4 \pm 1.0$ & $0.95(0.78-1.15)$ & 0.598 \\
\hline Triglyceride, mmol/L & $1.7 \pm 1.2$ & $1.6 \pm 1.0$ & $1.7 \pm 1.2$ & $0.94(0.78-1.13)$ & 0.511 \\
\hline $\mathrm{HDL}-\mathrm{C}, \mathrm{mmol} / \mathrm{L}$ & $1.1 \pm 0.3$ & $1.2 \pm 0.4$ & $1.1 \pm 0.3$ & $1.19(0.65-2.18)$ & 0.565 \\
\hline $\mathrm{LDL}-\mathrm{C}, \mathrm{mmol} / \mathrm{L}$ & $2.6 \pm 0.8$ & $2.6 \pm 0.8$ & $2.6 \pm 0.9$ & $0.92(0.72-1.17)$ & 0.482 \\
\hline $\mathrm{FBG}, \mathrm{mmol} / \mathrm{L}$ & $5.8 \pm 2.1$ & $6.1 \pm 2.7$ & $5.7 \pm 2.1$ & $1.07(0.99-1.16)$ & 0.099 \\
\hline $\mathrm{BUN}, \mathrm{mmol} / \mathrm{L}$ & $5.0 \pm 1.8$ & $5.7 \pm 1.9$ & $4.9 \pm 1.8$ & $1.17(1.08-1.27)$ & 0.001 \\
\hline UA, $10 \mu \mathrm{mol} / \mathrm{L}$ & $291.3 \pm 96.0$ & $301.6 \pm 114.1$ & $290.2 \pm 94.0$ & $1.01(0.99-1.03)$ & 0.296 \\
\hline$A L P, U / L$ & $77.6 \pm 25.6$ & $80.6 \pm 34.5$ & $77.2 \pm 24.5$ & $1.01(1.00-1.01)$ & 0.165 \\
\hline $\mathrm{BPC}, \times 10^{11} / \mathrm{L}$ & $194.4 \pm 58.3$ & $189.7 \pm 55.1$ & $194.8 \pm 58.6$ & $0.86(0.60-1.23)$ & 0.386 \\
\hline Leukocyte count, $\times 10^{9} / \mathrm{L}$ & $6.7 \pm 2.2$ & $7.2 \pm 2.8$ & $6.6 \pm 2.2$ & $1.09(1.01-1.17)$ & 0.024 \\
\hline
\end{tabular}

Abbreviations: BMI Body Mass Index, DM Diabetes Mellitus, AF Atrial Fibrillation, PVD Peripheral Vascular Disease, NIHSS National Institutes of Health Stroke Scale, SBP Systolic Blood Pressure, DBP Diastolic Blood Pressure, HLD-C High-Density Lipoprotein Cholesterol, LDL-C low-density lipoprotein cholesterol, FBG Fasting Blood Glucose, BUN Blood Urea Nitrogen, UA Uric Acid, ALP Alkaline Phosphatase, BPC Blood Platelet Count, Cl confidence internals, OR odds ratios, IQR interquartile range 
Table 3 Univariate logistic regression analysis of patients with or without all-cause mortality at 1 year after MIS

\begin{tabular}{|c|c|c|c|c|c|}
\hline Variables & $\begin{array}{l}\text { Overall } \\
1121\end{array}$ & $\begin{array}{l}\text { Death } \\
N=37(3.3 \%)\end{array}$ & $\begin{array}{l}\text { No death } \\
N=1084(96.7 \%)\end{array}$ & OR $(95 \% \mathrm{Cl})$ & $P$ value \\
\hline \multicolumn{6}{|l|}{ Demographic information } \\
\hline Age, median (IQR), year & $64.0(56.0-74.0)$ & $75.0(65.0-80.0)$ & $64.0(55.0-73.0)$ & $1.07(1.04-1.11)$ & 0.001 \\
\hline Male, n\% & $697(62.2)$ & $28(75.7)$ & $669(61.7)$ & $1.91(0.90-4.04)$ & 0.092 \\
\hline BMl on admission, $\mathrm{kg} / \mathrm{m}^{2}$ & $23.9(22.2-25.6)$ & $23.7(21.5-24.8)$ & $24.0(22.2-25.6)$ & $0.93(0.83-1.04)$ & 0.179 \\
\hline Education level, n (\%) & & & & & 0.494 \\
\hline elementary or below & $446(39.8)$ & $15(40.5)$ & $431(39.8)$ & Ref. & - \\
\hline middle school & $234(20.9)$ & $5(13.5)$ & $229(21.1)$ & $0.62(0.23-1.71)$ & 0.356 \\
\hline high school or above & $441(39.3)$ & $17(45.9)$ & $424(39.1)$ & $1.13(0.57-2.27)$ & 0.723 \\
\hline Medical insurance type, n (\%) & & & & & 0.944 \\
\hline Urban employees' medical insurance & $591(52.7)$ & $21(56.8)$ & $570(52.6)$ & Ref. & - \\
\hline New type rural cooperative medical system & $414(36.9)$ & $13(35.1)$ & $401(37.0)$ & $0.90(0.45-1.79)$ & 0.761 \\
\hline Commercial insurance & $2(0.2)$ & $0(0.0)$ & $2(0.2)$ & $0.00(0.00-\infty)$ & 0.997 \\
\hline Out-of-pocket medical & $114(10.2)$ & $3(8.1)$ & $111(10.2)$ & $0.74(0.22-2.47)$ & 0.622 \\
\hline \multicolumn{6}{|l|}{ Vascular risk factors } \\
\hline Hypertension, n (\%) & $768(68.5)$ & $22(59.5)$ & $746(68.8)$ & $0.66(0.34-1.28)$ & 0.219 \\
\hline $\mathrm{DM}, \mathrm{n}(\%)$ & $242(21.6)$ & $7(18.9)$ & $235(21.7)$ & $0.85(0.37-1.92)$ & 0.689 \\
\hline Dyslipidemia, n (\%) & $77(6.9)$ & $1(2.7)$ & $76(7.0)$ & $0.37(0.05-2.71)$ & 0.328 \\
\hline$A F, n(\%)$ & $54(4.8)$ & $4(10.8)$ & $50(4.6)$ & $2.39(0.85-6.75)$ & 0.099 \\
\hline Smoking, n (\%) & & & & & 0.272 \\
\hline Never smoking & $665(59.3)$ & $19(51.4)$ & $646(59.6)$ & Ref. & - \\
\hline smoking cessation & $169(15.1)$ & $9(24.3)$ & $160(14.8)$ & $1.92(0.87-4.24)$ & 0.107 \\
\hline Current smoking & $287(25.6)$ & $9(24.3)$ & $278(25.6)$ & $1.09(0.50-2.42)$ & 0.823 \\
\hline Moderate or heavy alcohol use, n (\%) & $271(24.2)$ & $6(16.2)$ & $265(24.4)$ & $0.59(0.25-1.42)$ & 0.241 \\
\hline Previous stroke, n (\%) & $297(26.5)$ & $14(37.8)$ & $283(26.1)$ & $1.73(0.89-3.35)$ & 0.108 \\
\hline PVD, n (\%) & $29(2.6)$ & $3(8.1)$ & $26(2.4)$ & $3.42(1.05-11.15)$ & 0.041 \\
\hline Pneumonia, n (\%) & $25(2.2)$ & $4(10.8)$ & $21(1.9)$ & $6.95(2.46-19.63)$ & 0.003 \\
\hline NIHSS score on admission & $1.0(0.0-2.0)$ & $2.0(0.0-3.0)$ & $1.0(0.0-2.0)$ & $1.25(0.95-1.65)$ & 0.108 \\
\hline SBP on admission, $\mathrm{mmHg}$ & $144.8 \pm 20.7$ & $146.2 \pm 21.2$ & $144.8 \pm 20.7$ & $1.00(0.99-1.02)$ & 0.652 \\
\hline DBP on admission, $\mathrm{mmHg}$ & $85.7 \pm 12.2$ & $85.3 \pm 13.7$ & $85.7 \pm 12.1$ & $1.00(0.97-1.02)$ & 0.853 \\
\hline Heart rate, bmp & $74.3 \pm 9.4$ & $77.0 \pm 7.9$ & $74.2 \pm 9.4$ & $1.03(1.00-1.06)$ & 0.061 \\
\hline \multicolumn{6}{|l|}{ Laboratory findings } \\
\hline Total cholesterol, mmol/L & $4.4 \pm 1.0$ & $4.2 \pm 0.7$ & $4.4 \pm 1.0$ & $0.79(0.56-1.12)$ & 0.191 \\
\hline Triglyceride, mmol/L & $1.7 \pm 1.2$ & $1.4 \pm 0.9$ & $1.7 \pm 1.2$ & $0.75(0.50-1.14)$ & 0.180 \\
\hline $\mathrm{HDL}-\mathrm{C}, \mathrm{mmol} / \mathrm{L}$ & $1.1 \pm 0.3$ & $1.1 \pm 0.3$ & $1.1 \pm 0.3$ & $0.96(0.33-2.76)$ & 0.939 \\
\hline $\mathrm{LDL}-\mathrm{C}, \mathrm{mmol} / \mathrm{L}$ & $2.6 \pm 0.8$ & $2.4 \pm 0.6$ & $2.6 \pm 0.9$ & $0.72(0.47-1.10)$ & 0.132 \\
\hline $\mathrm{FBG}, \mathrm{mmol} / \mathrm{L}$ & $5.8 \pm 2.1$ & $5.8 \pm 2.3$ & $5.8 \pm 2.1$ & $1.01(0.87-1.17)$ & 0.909 \\
\hline $\mathrm{BUN}, \mathrm{mmol} / \mathrm{L}$ & $5.0 \pm 1.8$ & $5.4 \pm 1.6$ & $5.0 \pm 1.8$ & $1.11(0.95-1.29)$ & 0.196 \\
\hline UA, $10 \mu \mathrm{mol} / \mathrm{L}$ & $291.3 \pm 96.0$ & $319.1 \pm 130.8$ & $290.3 \pm 94.5$ & $1.03(1.00-1.06)$ & 0.082 \\
\hline$A L P, U / L$ & $77.6 \pm 25.6$ & $87.2 \pm 43.7$ & $77.2 \pm 24.7$ & $1.01(1.00-1.02)$ & 0.012 \\
\hline $\mathrm{BPC}, \times 10^{11} / \mathrm{L}$ & $194.4 \pm 58.3$ & $181.6 \pm 54.1$ & $194.8 \pm 58.4$ & $0.67(0.37-1.22)$ & 0.187 \\
\hline $\mathrm{LC}, \times 10^{9} / \mathrm{L}$ & $6.7 \pm 2.2$ & $6.5 \pm 2.3$ & $6.7 \pm 2.2$ & $0.97(0.83-1.13)$ & 0.699 \\
\hline
\end{tabular}

Abbreviations: BMI Body Mass Index, DM Diabetes Mellitus, AF Atrial Fibrillation, PVD Peripheral Vascular Disease, NIHSS National Institutes of Health Stroke Scale, SBP Systolic Blood Pressure, DBP Diastolic Blood Pressure, HLD-C High-Density Lipoprotein Cholesterol, LDL-C low-density lipoprotein cholesterol, FBG Fasting Blood Glucose, BUN Blood Urea Nitrogen, UA Uric Acid, ALP Alkaline Phosphatase, BPC Blood Platelet Count, CI confidence internals, OR odds ratios, IQR interquartile range 
patients lost to follow-up, only medical insurance type differed between the two groups and there was no statistical difference in other variables (data not shown).

\section{Risk factors for outcomes at 1 year after MIS}

Multivariate logistic regression analysis was used to identify independent risk factors for stroke recurrence, disability, and all-cause mortality at 1 year after MIS. The results showed that age (odds ratio $[\mathrm{OR}]=1.04 ; 95 \%$ confidence interval $[\mathrm{CI}]=1.01-1.08 ; p=0.013)$, current smoking $(\mathrm{OR}=3.25 ; 95 \% \mathrm{CI}=1.33-7.93 ; p=0.010)$, and pneumonia $(\mathrm{OR}=6.80 ; 95 \% \mathrm{CI}=2.24-20.64 ; p=0.001)$ were independent risk factors for stroke recurrence at 1 year after MIS onset. The independent risk factors for stroke disability at 1 year were age $(\mathrm{OR}=1.09 ; 95 \% \mathrm{CI}=$ $1.06-1.12 ; p=0.001)$, pneumonia $(\mathrm{OR}=6.24 ; 95 \% \mathrm{CI}=$ $2.35-16.55 ; p=0.000)$, NIHSS score on admission (OR = $1.24 ; 95 \% \mathrm{CI}=1.01-1.53 ; p=0.036)$, and leukocyte count $(\mathrm{OR}=1.09 ; 95 \% \mathrm{CI}=1.00-1.20 ; p=0.048)$. Age $(\mathrm{OR}=$ 1.06; $95 \% \mathrm{CI}=1.03-1.11 ; p=0.001)$, pneumonia $(\mathrm{OR}=$ 4.48; $95 \% \mathrm{CI}=1.27-15.83 ; p=0.020)$, and ALP levels $(\mathrm{OR}=1.01 ; 95 \% \mathrm{CI}=1.00-1.02 ; p=0.023)$ were independent risk factors associated with all-cause mortality at 1 year after MIS onset (Table 4).

\section{Discussion}

This study is the largest stroke registration study to date in Xi'an, China. The results of the present study showed that a small number of patients with MIS experienced stroke recurrence, disability and all-cause mortality during the 1-year follow-up period. Nevertheless, these results are not optimistic. Hence, the risk factors associated with poor clinical outcomes at 1 year after MIS need to be further investigated. The results suggested that the risk factors associated with poor outcomes at 1 year after MIS stroke (i.e., recurrence, disability, and allcause mortality) were not entirely consistent in Xi'an, China. Therefore, clinicians should apply early prevention strategies on an individual basis.The results further showed that the prevalence of stroke recurrence at 1 year after MIS in the Xi'an region of China was 3.4\%, which was lower than the prevalence of $13.2 \%$ at 1 year and that of $9.8 \%$ at 3 months reported by the China National Stroke Registry (CNSR) study [6, 15]; lower than the stroke recurrence prevalence of $7.6 \%$ in the Clopidogrel in high-risk patients with the Acute Non-disabling Cerebrovascular Events (CHANCE) study [16]; also lower than the stroke recurrence prevalence of $6.1 \%$ by analysis of the Korean Multicenter Stroke Registry [17]; but close to the stroke recurrence prevalence of 3.7\% recently reported by the TIAregistry.org project [18]. Besides, our data also revealed lower mortality (3.3\% vs. $6.3 \%)$ and disability $(9.3 \%$ vs. $17 \%)$ at 1 year as compared with the CNSR study [13], but with a comparable mortality (3.3\% vs. $4.1 \%)$ to the Korean Multicenter Stroke Registry study at 1 year after MIS [17]. These results suggested that the clinical outcomes of patients with MIS may differ among countries and regions. In addition to the differences in study designs, the prevalence of clinical outcomes may also be related to geographical environments, daily habits, economic status, and disease prevention measures, indicating the importance of studies of regional stroke registries.

There are several potential reasons for the lower prevalence of stroke recurrence, disability, and all-cause mortality at 1 year in the present study. First, there were notable differences in the clinical characteristics between our study and previous studies. As compared to the CNSR study [6], patients included in the present study had lower prevalence of hypertension (68.5\% vs. $73.6 \%)$,

Table 4 Multivariable logistic regression analysis of independent risk factors associated with stroke recurrence, disability and allcause mortality at 1 year after MIS onset

\begin{tabular}{|c|c|c|c|c|c|c|}
\hline Variables & $\begin{array}{l}\text { Stroke recurrence } \\
\text { OR }(95 \% \mathrm{Cl})\end{array}$ & $P$ value & $\begin{array}{l}\text { Stroke disability } \\
\text { OR }(95 \% \mathrm{Cl})\end{array}$ & $P$ value & $\begin{array}{l}\text { Death } \\
\text { OR }(95 \% \mathrm{Cl})\end{array}$ & $P$ value \\
\hline Age & $1.04(1.01-1.08)$ & 0.013 & $1.09(1.06-1.12)$ & 0.001 & $1.06(1.03-1.11)$ & 0.001 \\
\hline Moderate or heavy Alcohol use & $0.36(0.13-1.03)$ & 0.056 & $0.45(0.19-1.05)$ & 0.064 & $0.31(0.22-1.00)$ & 0.055 \\
\hline Current smoking & $3.25(1.33-7.93)$ & 0.010 & - & - & $2.06(0.78-5.45)$ & 0.146 \\
\hline Previous stroke & $1.89(0.91-3.91)$ & 0.087 & $1.28(0.76-2.17)$ & 0.356 & - & - \\
\hline PVD & $1.80(0.37-8.77)$ & 0.465 & $0.66(0.14-3.14)$ & 0.601 & $2.20(0.44-10.88)$ & 0.334 \\
\hline Pneumonia & $6.80(2.24-20.64)$ & 0.001 & $6.24(2.35-16.55)$ & $<0.001$ & $4.48(1.27-15.83)$ & 0.020 \\
\hline ALP & - & - & - & - & $1.01(1.00-1.02)$ & 0.023 \\
\hline NIHSS score on admission & $0.88(0.65-1.20)$ & 0.420 & $1.24(1.01-1.53)$ & 0.036 & $1.08(0.80-1.48)$ & 0.607 \\
\hline BUN & - & - & $1.11(0.99-1.24)$ & 0.088 & - & - \\
\hline Leukocyte count & $1.03(0.90-1.19)$ & 0.651 & $1.09(1.00-1.20)$ & 0.048 & $0.94(0.80-1.11)$ & 0.465 \\
\hline
\end{tabular}

Stroke disability was defined as modified Rankin Scale $\geq 3$. "-"not included in the multivariate analysis Abbreviations: OR Odd Ratio, Cl Confidence Internal, PVD Peripheral vascular disease, ALP Alkaline phosphatase, BUN Blood urea nitrogen; Adjusted for age, education level, moderate or heavy alcohol, current smoking, previous stroke, PVD, Pneumonia, ALP, NIHSS score on admission, BUN and Leukocyte count 
DM (21.6\% vs. $27.3 \%)$, dyslipidemia (6.9\% vs. $11.8 \%)$, atrial fibrillation $(4.8 \%$ vs. $5.8 \%)$, and previous stroke (26.5\% vs. $31.1 \%$ ), as well as lower NIHSS scores on admission (median, 1 vs. 2). Hence, the prevalence of risk factors for clinical outcomes of stroke in this region was relatively lower, which may be related to the better preventive measures and lifestyles in the Xi'an region, as compared with other regions. Second, differences in study designs and regions may have led to the differences in results. The CNSR study was a nationwide survey $[6,15]$ and, thus, did not represent the status quo. The datas assessed in the present study were collected from four tertiary grade A hospitals in the Xi'an region, which corresponding to the lower valley of the Wei River in the Guanzhong Plain in northern China. The relatively lower prevalence of poor outcomes may be due to more standardized regimens for the diagnosis, treatment, and prevention of secondary stroke than those in the CNSR study, which included primary, secondary, and tertiary hospitals. Other potential reasons for the lower prevalence of poor outcomes in the present study might be that most of the patients resided in urban areas of the Xi'an region and more than $90 \%$ had medical insurance.

Risk factors affecting the 1-year outcomes (i.e., stroke recurrence, disability, and all-cause mortality) after MIS in the Xi'an region of China were investigated. In the present and previous studies, age was identified as an independent risk factor for stroke recurrence, disability, and all-cause mortality at 1 year after MIS [19, 20]. Hence, older patients should be closely monitored for various indicators and early detection and treatment.

In addition, pneumonia was identified as an independent risk factor for stroke recurrence, disability, and allcause mortality at 1 year after MIS in the Xi'an region, similar with the findings of previous studies [21, 22]. Pneumonia is closely related to dysphagia caused by stroke [22], suggesting that treatment for swallow difficulties after stroke must be improved in the Xi'an region. So, clinicians should promptly evaluate patients with dysphagia and initiate swallow rehabilitation, dietary guidance, and education of dysphagia in order to reduce the prevalence of pneumonia after MIS and improve treatment outcomes.

Similar to previous studies [23-25], current smoking was found to be an independent risk factor associated with 1-year stroke recurrence after MIS. After stroke, persistent smoking increases the risk of stroke recurrence. There exists a dose-response relationship between smoking quantity and the risk of stroke recurrence [24, 25] because smoking increases the short-term risk of stroke by promoting thrombosis and reducing cerebral blood flow via arterial vasoconstriction [26, 27]. Therefore, it is important to control smoking among MIS patients.
In this study, an elevation in ALP levels was an independent risk factor for all-cause mortality, in accordance with the findings of previous studies [28-30]. Elevated ALP was related with an increased risk of all-cause mortality in patients with end-stage renal disease $[28,29]$ and as an independent predictor of poor outcomes of patients with preserved kidney function in the CNSR study [30]. As a possible explanation, serum ALP has been implicated in the pathogenesis of vascular calcification and subclinical atherosclerosis [31, 32]. Vascular calcification plays a significant role in the process of atherosclerosis and also leads to increase vascular stiffness and reduce vascular compliance. So, clinicians should pay more attention to ALP levels in patients with MIS, as early detection and intervention may reduce the risk of death within 1 year after stroke.

In the present study, the NIHSS score and leukocyte count on admission were identified as risk factors for stroke disability at 1 year after MIS, which was consistent with the findings of previous results [33-36]. A higher NIHSS score indicates severe neurological impairment. Because there is no specific treatment for cerebral function injury caused by stroke, the outcomes of the majority of patients with severe neurological impairment were generally poor. Previous studies have reported that a high leukocyte count in the early stage of stroke was closely related to the severity of stroke and co-infection, which led to aggravation of stroke and subsequent disability [37, 38]. However, an elevated leukocyte count in the early stage of stroke may not necessarily be caused by infection, thus the clinician should assess the presence of co-infections. For non-infectious stroke, the patient's family members should be informed of a potentially poor outcome as early as possible. Early prevention and treatment of digestive tract ulcers and acute brain-heart syndrome may be hampered by a state of stress.

In addition, multivariate analysis showed that alcohol seemed to be a protective factor. The possible explanation is that we only recorded whether the alcohol used or not, but did not record the amount of alcohol consumed. However, based on the clinical characteristics, the mean age was higher in the patients with MIS who had adverse outcomes (include stroke recurrence, disability, all-cause mortality) at 1-year follow up. This may be due to the fact that most of the patients were in good health before the onset, more patients may have previously drunk alcohol. This phenomenon may lead to the tendency of alcohol consumption to be a protective factor in our multivariate analysis, but the result was not statistically significant.

There were some limitations to this study that should be addressed. First, the four hospitals participating in this study were not selected at random, thus there was 
potential for selection bias when evaluated the real burden of the disease in the Xi'an region of China. In addition, all the participating hospitals were level 3 firstclass hospitals that may not necessarily represent the status quo of MIS treatment in community hospitals. Second, the focus of this study was the influence of risk factors on admission and during hospitalization on 1year outcomes, so potential factors after discharge were not analyzed. Third, the data obtained from cerebrovascular and neurological imaging in this study were incomplete, so there were a lack of image-related risk factors, such as infarct volume and infarct location. Forth, in this study, 131 (10.5\%) patients were lost to follow-up at 1 year after MIS. However, the sensitivity analysis showed that the patients lost to follow-up in this study were nearly random, which did not affect the results.

\section{Conclusions}

In conclusion, the 1-year outcomes of MIS in Xi'an region of China were not optimistic, especially high prevalence of disability. The present study indicated several risk factors affecting the 1-year outcomes of MIS in Xi'an region of China. Of note, age and pneumonia were the common independent risk factors for 1-year stroke recurrence, disability, and all-cause mortality.

\section{Abbreviations \\ AF: atrial fibrillation; ALP: alkaline phosphatase; BMI: body mass index; BPC: blood platelet count; BUN: blood urea nitrogen; CHANCE: clopidogrel in high-risk patients with acute non-disabling cerebrovascular events; Cl: confidence internal; CNSR: China national stroke registry; CT: computed tomography; DBP: diastolic blood pressure; DM: diabetes mellitus; FBG: fasting blood glucose; HLD-C: high-density lipoprotein cholesterol; IQR: interquartile range; LDL-C: low-density lipoprotein cholesterol; MIS: minor ischemic stroke; MRI: magnetic resonance imaging; NIHS S: National Institutes of Health stroke scale; OR: odds ratio; PVD: peripheral vascular disease; SBP: systolic blood pressure; UA: uric acid}

\section{Acknowledgements}

We are grateful to all the imaging and laboratory technicians, medical staffs, and nurses from the participating hospitals. Also, we thank International Science Editing (http://www.internationalscienceediting.com) for editing this manuscript.

\section{Authors' contributions}

ZZL and SDW planned and designed the study. WJL, QLL, JW, PL, YLS, QW, and GZL contributed to data acquisition and interpretation. ZZL analyzed the data. ZZL and SDW were primarily responsible for writing the paper. XML and FW revised the manuscript for important intellectual content. All authors read and approved the final version of the manuscript.

\section{Funding}

Financial Disclosure: This work was supported by the Science and Technology Program of Shaanxi Province (grant no. 2017SF163), the Science and Technology Plan Major Project of Xi'an city [grant no. 201805104YX12SF38(2)], and the Scientific Research Project of the Xi'an Health Commission (grant nos. 2020 ms03 and 2020yb05). The funding body had no role in the design of the study and collection, analysis, and interpretation of data and in writing the manuscript.

\section{Availability of data and materials}

The datasets used and/or analyzed during the current study are available from the corresponding author on reasonable request.

\section{Ethics approval and consent to participate}

The study protocol was approved by the ethics committees of the First Hospital of Xi'an and conducted in accordance with the ethical principles for medical research involving human subjects as described in the Declaration of Helsinki. Written informed consent was obtained from all patients participating in this study.

\section{Consent for publication}

Not applicable.

\section{Competing interests}

The authors have no competing interests to declare.

\section{Author details}

'The First Affiliated Hospital of Northwest University, Xi'an 710069, Shaanxi, China. ${ }^{2}$ Department of Neurology, The First Hospital of Xi'an, No.30, Fenxiang Road, South Street, Xi'an 710002, Shaanxi, China. ${ }^{3}$ College of life Science, Northwest University, Xi'an 710069, Shaanxi, China.

Received: 25 June 2020 Accepted: 7 October 2020

Published online: 20 October 2020

\section{References}

1. Collaborators GBDS. Global, regional, and national burden of stroke, 19902016: a systematic analysis for the global burden of disease study 2016. Lancet Neurol. 2019;18(5):439-58.

2. Liu L, Wang D, Wong KS, Wang Y. Stroke and stroke care in China: huge burden, significant workload, and a national priority. Stroke. 2011;42(12): 3651-4.

3. Yang G, Wang Y, Zeng $Y$, Gao GF, Liang $X$, Zhou M, et al. Rapid health transition in China, 1990-2010: findings from the global burden of disease study 2010. Lancet. 2013;381(9882):1987-2015.

4. Fischer U, Baumgartner A, Arnold M, Nedeltchev K, Gralla J, De Marchis GM, et al. What is a minor stroke? Stroke. 2010;41(4):661-6.

5. Yu AY, Hill MD, Coutts SB. Should minor stroke patients be thrombolyzed? A focused review and future directions. Int I Stroke. 2015;10(3):292-7.

6. Wu L, Wang A, Wang X, Zhao X, Wang C, Liu L, et al. Factors for short-term outcomes in patients with a minor stroke: results from China National Stroke Registry. BMC Neurol. 2015;15:253.

7. Ferrari J, Knoflach M, Kiechl S, Willeit J, Schnabl S, Seyfang L, et al. Early clinical worsening in patients with TIA or minor stroke: the Austrian stroke unit registry. Neurology. 2010;74(2):136-41.

8. Stroke--1989. Recommendations on stroke prevention, diagnosis, and therapy. Report of the WHO Task Force on Stroke and other Cerebrovascular Disorders. Stroke. 1989;20(10):1407-31.

9. Goldstein LB, Bertels C, Davis JN. Interrater reliability of the NIH stroke scale. Arch Neurol. 1989;46(6):660-2.

10. Zou FTY, Wu WG. Design and implementation of management system for stroke data mining based on non-structured electronic medical record. China Digital Medicine. 2015;10(2):41-4

11. Brott T, Adams HP Jr, Olinger CP, Marler JR, Barsan WG, Biller J, et al. Measurements of acute cerebral infarction: a clinical examination scale. Stroke. 1989;20(7):864-70.

12. Jia Q, Zhao X, Wang C, Wang Y, Yan Y, Li H, et al. Diabetes and poor outcomes within 6 months after acute ischemic stroke: the China National Stroke Registry. Stroke. 2011;42(10):2758-62.

13. Asplund K, Tuomilehto J, Stegmayr B, Wester PO, Tunstall-Pedoe H. Diagnostic criteria and quality control of the registration of stroke events in the MONICA project. Acta Med Scand Suppl. 1988;728:26-39.

14. Quinn TJ, Dawson J, Walters MR, Lees KR. Exploring the reliability of the modified Rankin scale. Stroke. 2009;40(3):762-6.

15. Tan Y, Pan Y, Liu L, Wang Y, Zhao X. One-year outcomes and secondary prevention in patients after acute minor stroke: results from the China National Stroke Registry. Neurol Res. 2017;39(6):484-91.

16. Wang G, Jing J, Pan Y, Meng X, Zhao X, Liu L, et al. Does all single infarction have lower risk of stroke recurrence than multiple infarctions in minor stroke? BMC Neurol. 2019;19(1):7. 
17. Park HK, Kim BJ, Han MK, Park JM, Kang K, Lee SJ, et al. One-year outcomes after minor stroke or high-risk transient ischemic attack: Korean multicenter stroke registry analysis. Stroke. 2017;48(11):2991-8.

18. Amarenco P, Lavallee PC, Labreuche J, Albers GW, Bornstein NM, Canhao P, et al. One-year risk of stroke after transient ischemic attack or minor stroke. N Engl J Med. 2016;374(16):1533-42.

19. Knoflach M, Matosevic B, Rucker M, Furtner M, Mair A, Wille G, et al. Functional recovery after ischemic stroke--a matter of age: data from the Austrian stroke unit registry. Neurology. 2012;78(4):279-85.

20. Feng W, Hendry RM, Adams RJ. Risk of recurrent stroke, myocardial infarction, or death in hospitalized stroke patients. Neurology. 2010;74(7): 588-93

21. Hannawi Y, Hannawi B, Rao CP, Suarez JI, Bershad EM. Stroke-associated pneumonia: major advances and obstacles. Cerebrovasc Dis. 2013;35(5):430-43.

22. Eltringham SA, Kilner K, Gee M, Sage K, Bray BD, Pownall S, et al. Impact of dysphagia assessment and management on risk of stroke-associated pneumonia: a systematic review. Cerebrovasc Dis. 2018;46(3-4):99-107.

23. McNally JS, McLaughlin MS, Hinckley PJ, Treiman SM, Stoddard GJ, Parker DL, et al. Intraluminal thrombus, intraplaque hemorrhage, plaque thickness, and current smoking optimally predict carotid stroke. Stroke. 2015;46(1):84-90.

24. Markidan J, Cole JW, Cronin CA, Merino JG, Phipps MS, Wozniak MA, et al. Smoking and risk of ischemic stroke in young men. Stroke. 2018;49(5):1276-8.

25. Chen J, Li S, Zheng K, Wang H, Xie Y, Xu P, et al. Impact of smoking status on stroke recurrence. J Am Heart Assoc. 2019;8(8):e011696.

26. Ambrose JA, Barua RS. The pathophysiology of cigarette smoking and cardiovascular disease: an update. J Am Coll Cardiol. 2004:43(10):1731-7.

27. Rogers RL, Meyer JS, Judd BW, Mortel KF. Abstention from cigarette smoking improves cerebral perfusion among elderly chronic smokers. JAMA. 1985;253(20):2970-4.

28. Waziri B, Duarte $R$, Naicker $S$. High serum alkaline phosphatase, Hypercalcaemia, race, and mortality in south African maintenance Haemodialysis patients. Int J Nephrol. 2017;2017:2795432.

29. Wannamethee SG, Sattar N, Papcosta O, Lennon L, Whincup PH. Alkaline phosphatase, serum phosphate, and incident cardiovascular disease and total mortality in older men. Arterioscler Thromb Vasc Biol. 2013;33(5):1070-6.

30. Zong L, Wang X, Li Z, Zhao X, Liu L, Li H, et al. Alkaline phosphatase and outcomes in patients with preserved renal function: results from China National Stroke Registry. Stroke. 2018;49(5):1176-82.

31. Cheung BM, Ong KL, Wong LY. Elevated serum alkaline phosphatase and peripheral arterial disease in the United States National Health and nutrition examination survey 1999-2004. Int J Cardiol. 2009;135(2):156-61.

32. Schutte R, Huisman HW, Malan L, van Rooyen JM, Smith W, Glyn MC, et al. Alkaline phosphatase and arterial structure and function in hypertensive African men: the SABPA study. Int J Cardiol. 2013;167(5):1995-2001.

33. Liu $X, L v Y$, Wang B, Zhao G, Yan $Y, X u$ D. Prediction of functional outcome of ischemic stroke patients in Northwest China. Clin Neurol Neurosurg. 2007;109(7):571-7

34. Villringer K, Florczak-Rzepka M, Grittner U, Brunecker P, Tepe H, Nolte $\mathrm{CH}_{\text {, }}$ et al. Characteristics associated with outcome in patients with first-ever posterior fossa stroke. Eur J Neurol. 2018;25(6):818-24.

35. Kim J, Song TJ, Park JH, Lee HS, Nam CM, Nam HS, et al. Different prognostic value of white blood cell subtypes in patients with acute cerebral infarction. Atherosclerosis. 2012;222(2):464-7.

36. Wu TH, Chien KL, Lin HJ, Hsu HC, Su TC, Chen MF, et al. Total white blood cell count or neutrophil count predict ischemic stroke events among adult Taiwanese: report from a community-based cohort study. BMC Neurol. 2013;13:7.

37. Minic GA. Leucocyte count indicates carotid plaque instability in stroke patients. Vojnosanit Pregl. 2016;73(6):515-25

38. Rezende SM, Lijfering WM, Rosendaal FR, Cannegieter SC. Hematologic variables and venous thrombosis: red cell distribution width and blood monocyte count are associated with an increased risk. Haematologica. 2014 99(1):194-200

\section{Publisher's Note}

Springer Nature remains neutral with regard to jurisdictional claims in published maps and institutional affiliations.

\section{Ready to submit your research? Choose BMC and benefit from:}

- fast, convenient online submission

- thorough peer review by experienced researchers in your field

- rapid publication on acceptance

- support for research data, including large and complex data types

- gold Open Access which fosters wider collaboration and increased citations

- maximum visibility for your research: over $100 \mathrm{M}$ website views per year

At BMC, research is always in progress.

Learn more biomedcentral.com/submissions 